\title{
@creative
}

Also available at http://amc-journal.eu

ISSN 1855-3966 (printed edn.), ISSN 1855-3974 (electronic edn.)

ARS MATHEMATICA CONTEMPORANEA 12 (2017) 37-50

\section{On skew Heyting algebras}

\author{
Karin Cvetko-Vah \\ University of Ljubljana, Faculty of Mathematics and Physics, \\ Jadranska 19, Ljubljana, Slovenia
}

Received 29 October 2014, accepted 8 April 2016, published online 21 April 2016

\begin{abstract}
In the present paper we generalize the notion of a Heyting algebra to the non-commutative setting and hence introduce what we believe to be the proper notion of the implication in skew lattices. We list several examples of skew Heyting algebras, including Heyting algebras, dual skew Boolean algebras, conormal skew chains and algebras of partial maps with poset domains.
\end{abstract}

Keywords: Skew lattices, Heyting algebras, non-commutative algebra, intuitionistic logic.

Math. Subj. Class.: 06F35, 03G27

\section{Introduction}

Non-commutative generalizations of lattices were introduced by Jordan [11] in 1949. The current approach to such objects began with Leech's 1989 paper on skew lattices [13]. Similarly, skew Boolean algebras are non-commutative generalizations of Boolean algebras. In 1936 Stone proved that each Boolean algebra can be embedded into a field of sets [20]. Likewise, Leech showed in [14, 15] that each right-handed skew Boolean algebra can be embedded into a generic skew Boolean algebra of partial functions from a given set to the codomain $\{0,1\}$. Bignall and Leech [5] showed that skew Boolean algebras play a central role in the study of discriminator varieties.

Though not using categorical language, Stone essentially proved in [20] that the category of Boolean algebras and homomorphisms is dual to the category of Boolean topological spaces and continuous maps. Generalizations of this result within the commutative setting yield Priestley duality [16, 17] between bounded distributive lattices and Priestley spaces, and Esakia duality [9] between Heyting algebras and Esakia spaces. (See [4] for details.) In a recent paper [10] on Esakia's work, Gehrke showed that Heyting algebras may be understood as those distributive lattices for which the embedding into their Booleanisation has a right adjoint. A recent line of research generalized the results of Stone and

E-mail address: karin.cvetko@fmf.uni-lj.si (Karin Cvetko-Vah) 
Priestley to the non-commutative setting. By results in [1] and [12], any skew Boolean algebra is dual to a sheaf of rectangular bands over a locally-compact Boolean space. A further generalization given in [2] showed that any strongly distributive skew lattice (as defined below) is dual to a sheaf (of rectangular bands) over a locally compact Priestley space.

While Boolean algebras provide algebraic models of classical logic, Heyting algebras provide algebraic models of intuitionistic logic. In the present paper we introduce the notion of a skew Heyting algebra. In passing to the non-commutative setting one needs to sacrifice either the top or the bottom of the algebra in order not to end up in the commutative setting. In the previous papers [1], [12] and [2] algebras with bottoms were considered, and hence the notion of distributivity was generalized to the notion of so-called strong distributivity. If one tried to define an implication operation in the setting of strongly distributive skew lattices with a bottom as a right adjoint to conjunction, that would force the skew lattice to also possess a top and hence be commutative, resulting in a usual Heyting algebra. In order to define implication in the skew lattice setting we consider the $\vee-\wedge$ duals of strongly distributive skew lattices with a bottom, namely, the co-strongly distributive skew lattices with a top. That is not surprising as a top plays a crucial role in logic. The category of co-strongly distributive skew lattices with a top is, of course, isomorphic to the category of strongly distributive skew lattices with a bottom. In choosing co-strongly distributive skew lattices with a top we follow the path paved by Bignall and Spinks in [6], and by Spinks and Veroff in [19] where dual skew Boolean algebras were introduced. For further reading on implications in skew Boolean algebras and their algebraic duals, see [7].

After reviewing some preliminary definitions and concepts in Section 2, in the next section we introduce the notion of a skew Heyting algebra, prove that such algebras form a variety and show that the maximal lattice image of a skew Heyting algebra is a generalized Heyting algebra (possibly without a bottom). Indeed, a co-strongly distributive skew lattice with a top is the reduct of a skew Heyting algebra, if and only if its maximal lattice image forms a generalized Heyting algebra. (See Theorem 3.5.) This leads to a number of useful corollaries and examples. We finish with Section 4 where we explore the consequences of our results to duality theory, and describe how skew Heyting algebras correspond to sheaves over local Esakia spaces.

\section{Preliminaries}

A skew lattice is an algebra $\mathbf{S}=(S ; \wedge, \vee)$ of type $(2,2)$ such that $\wedge$ and $\vee$ are both idempotent and associative and they satisfy the following absorption laws:

$$
x \wedge(x \vee y)=x=x \vee(x \wedge y) \text { and }(x \wedge y) \vee y=y=(x \vee y) \wedge y
$$

These identities are collectively equivalent to the pair of equivalences: $x \wedge y=x \Leftrightarrow x \vee y=$ $y$ and $x \wedge y=y \Leftrightarrow x \vee y=x$.

On a skew lattice $\mathbf{S}$ one can define the natural partial order by stating that $x \leq y$ if and only if $x \vee y=y=y \vee x$, or equivalentely $x \wedge y=z=y \wedge x$, and the natural preorder by $x \preceq y$ if and only if $y \vee x \vee y=y$, or equivalentely $x \wedge y \wedge x=x$. Green's equivalence relation $\mathcal{D}$ is then defined by

$$
x \mathcal{D} y \text { if and only if } x \preceq y \text { and } y \preceq x \text {. }
$$


Lemma 2.1. ([8]). For elements $x$ and $y$ elements of a skew lattice $\mathbf{S}$ the following are equivalent:

(i) $x \leq y$,

(ii) $x \vee y \vee x=y$,

(iii) $y \wedge x \wedge y=x$.

Leech's First Decomposition Theorem for skew lattices states that the relation $\mathcal{D}$ is a congruence on a skew lattice $\mathbf{S}, \mathbf{S} / \mathcal{D}$ is the maximal lattice image of $\mathbf{S}$, and each congruence class is a maximal rectangular skew lattice in $S$ [13]. Rectangular skew lattices are characterized by $x \wedge y \wedge z=x \wedge z$, or equivalentely $x \vee y \vee z=x \vee z$. We denote the $\mathcal{D}$-class containing an element $x$ by $\mathcal{D}_{x}$.

It was proved in [13] that a skew lattice always forms a regular band for either of the operations $\wedge, \vee$, i.e. it satisfies the identities

$$
x \wedge u \wedge x \wedge v \wedge x=x \wedge u \wedge v \wedge x \text { and } x \vee u \vee x \vee v \vee x=x \vee u \vee v \vee x
$$

A skew lattice with top is an algebra $(S ; \wedge, \vee, 1)$ of type $(2,2,0)$ such that $(S ; \wedge, \vee)$ is a skew lattice and $x \vee 1=1=1 \vee x$, or equivalently $x \wedge 1=x=1 \wedge x$, holds for all $x \in S$. A skew lattice with bottom is defined dually and the bottom, if it exists, is usually denoted by 0 .

Furthermore, a skew lattice is called strongly distributive if it satisfies the following identities:

$$
x \wedge(y \vee z)=(x \wedge y) \vee(x \wedge z) \text { and }(x \vee y) \wedge z=(x \wedge z) \vee(y \wedge z)
$$

and it is called co-strongly distributive if it satisfies the identities:

$$
x \vee(y \wedge z)=(x \vee y) \wedge(x \vee z) \text { and }(x \wedge y) \vee z=(x \vee z) \wedge(y \vee z)
$$

If a skew lattice $\mathbf{S}$ is either strongly distributive or co-strongly distributive then $\mathbf{S}$ is distributive in that it satisfies the identities

$x \wedge(y \vee z) \wedge x=(x \wedge y \wedge x) \vee(x \wedge z \wedge x)$ and $x \vee(y \wedge z) \vee x=(x \vee y \vee x) \wedge(x \vee z \vee x)$

A skew lattice $\mathbf{S}$ that is jointly strongly distributive and co-strongly distributive is $b i$ normal, i.e. $\mathbf{S}$ factors as a direct product of a lattice $\mathbf{L}$ and a rectangular skew lattice $\mathbf{B}$, $\mathbf{S} \cong \mathbf{L} \times \mathbf{B}$, with $\mathbf{L}$ in this case being distributive. (See [15] and [18].)

Applying duality to a result of Leech [15], it follows that a skew lattice $S$ is co-strongly distributive if and only if $\mathbf{S}$ is jointly:

- quasi-distributive: the maximal lattice image $\mathbf{S} / \mathcal{D}$ is a distributive lattice,

- symmetric: $x \wedge y=y \wedge x$ if and only if $x \vee y=y \vee x$, and

- conormal: $x \vee y \vee z \vee w=x \vee z \vee y \vee w$.

If a skew lattice is conormal then given any $u \in S$ the set

$$
u \uparrow=\{u \vee x \vee u \mid x \in S\}=\{x \in S \mid u \leq x\}
$$

forms a (commutative) lattice for the induced operations $\wedge$ and $\vee$, cf. [15].

The following lemma is the dual of a well known result in skew lattice theory. 
Lemma 2.2. Let $\mathbf{S}$ be a conormal skew lattice and let $A$ and $B$ be $\mathcal{D}$-classes such that $B \leq A$ holds in the lattice $\mathbf{S} / \mathcal{D}$. Given $b \in B$ there exists a unique $a \in A$ such that $b \leq a$.

Proof. First the uniqueness. If $a$ and $a^{\prime}$ both satisfy the desired property then by Lemma 2.1 we have $a=b \vee a \vee b$ and likewise $a^{\prime}=b \vee a^{\prime} \vee b$. Now, using idempotency of $\vee$, conormality and the fact that $a \mathcal{D} a^{\prime}$ we obtain:

$$
\begin{aligned}
a=b \vee a \vee b=b \vee a \vee a^{\prime} \vee a & \vee b= \\
b & \vee a \vee a^{\prime} \vee b=b \vee a^{\prime} \vee a \vee a^{\prime} \vee b=b \vee a^{\prime} \vee b=a^{\prime} .
\end{aligned}
$$

To prove the existence of $a$ take any $x \in A$ and set $a=b \vee x \vee b$. Then $a \in A$ and using the idempotency of $\vee$ we get:

$$
b \vee a \vee b=b \vee(b \vee x \vee b) \vee b=b \vee x \vee b=a
$$

which implies $b \leq a$.

An important class of strongly distributive skew lattices that have a bottom is the class of skew Boolean algebras where by a skew Boolean algebra we mean an algebra $\mathbf{S}=$ $(S ; \wedge, \vee, \backslash, 0)$ where $(S ; \wedge, \vee, 0)$ is a strongly distributive skew lattice with bottom 0 , and $\backslash$ is a binary operation on $S$ such that both $(x \wedge y \wedge x) \vee(x \backslash y)=x=(x \backslash y) \vee(x \wedge y \wedge x)$ and $(x \wedge y \wedge x) \wedge(x \backslash y)=0=(x \backslash y) \wedge(x \wedge y \wedge x)$. Given any element $u$ of a skew Boolean algebra $\mathbf{S}$ the set

$$
u \downarrow=\{u \wedge x \wedge u \mid x \in S\}=\{x \in S \mid x \leq u\}
$$

is a Boolean algebra with top $u$ and with $u \backslash x$ being the complement of $u \wedge x \wedge u$ in $u \downarrow$.

Recall that a Heyting algebra is an algebra $\mathbf{H}=(H ; \wedge, \vee, \rightarrow, 1,0)$ such that $(H, \wedge, \vee$, $1,0)$ is a bounded distributive lattice that satisfies the condition:

(HA) $x \wedge y \leq z$ iff $x \leq y \rightarrow z$.

Stated otherwise, $\forall y, z \in H$ the sublattice $\{x \in H \mid x \wedge y \leq z\}$ is nonempty and contains a top element to be denoted by $y \rightarrow z$. Thus, given a bounded distributive lattice $(H ; \wedge, \vee, 1,0)$, if a binary operation $\rightarrow$ exists that makes $(H ; \wedge, \vee, \rightarrow, 1,0)$ a Heyting algebra, then it is unique because it is already there implicitly. Indeed, given two isomorphic lattices, if either is the lattice reduct of a Heyting algebra then so is the other, and both are isomorphic as Heyting algebras.

Equivalently, (HA) can be replaced by the following set of identities:

(H1) $(x \rightarrow x)=1$,

(H2) $x \wedge(x \rightarrow y)=x \wedge y$,

(H3) $y \wedge(x \rightarrow y)=y$,

(H4) $x \rightarrow(y \wedge z)=(x \rightarrow y) \wedge(x \rightarrow z)$.

Lemma 2.3. In any Heyting algebra, $x \rightarrow y=(x \vee y) \rightarrow y$.

A generalized Heyting algebra is an algebra $\mathbf{A}=(A ; \wedge, \vee, \rightarrow, 1)$ such that the reduct $(A, \wedge, \vee, 1)$ is a distributive lattice with top 1 , and condition (HA) holds. If it also has a bottom, it is a Heyting algebra. In general, each upset $u \uparrow$ forms a Heyting algebra. The identities above also characterize this more general class of algebras, which are often called Brouwerian algebras. 


\section{Skew Heyting algebras}

A skew Heyting lattice is an algebra $\mathbf{S}=(S ; \wedge, \vee, 1)$ of type $(2,2,0)$ such that:

- $(S ; \wedge, \vee, 1)$ is a co-strongly distributive skew lattice with top 1 . Each upset $u$ sup is thus a bounded distributive lattice.

- for any $u \in S$ an operation $\rightarrow_{u}$ can be defined on $u \uparrow$ such that $\left(u \uparrow ; \wedge, \vee, \rightarrow_{u}, 1, u\right)$ is a Heyting algebra with top 1 and bottom $u$.

Given a skew Heyting lattice $\mathbf{S}$, define $\rightarrow$ on $S$ by setting

$$
x \rightarrow y=(y \vee x \vee y) \rightarrow_{y} y .
$$

A skew Heyting algebra is an algebra $\mathbf{S}=(S ; \wedge, \vee, \rightarrow, 1)$ of type $(2,2,2,0)$ such that $(S ; \wedge, \vee, 1)$ is a skew Heyting lattice and $\rightarrow$ is the implication thus induced. A sense of global coherence for $\rightarrow$ on $S$ is given by:

Lemma 3.1. Let $\mathbf{S}$ be a skew Heyting lattice with $\rightarrow$ as defined above and let $x, y, u \in S$ be such that both $x, y \in u \uparrow$ hold. Then $x \rightarrow y=x \rightarrow_{u} y$.

Proof. As $x$ and $y$ both lie in $u \uparrow$, they commute. By the definition of $\rightarrow, x \rightarrow y=$ $(x \vee y) \rightarrow_{y} y \geq y$ by (H3). On the other hand, since $\rightarrow_{u}$ is the Heyting implication in the Heyting algebra $u \uparrow$ it follows that $x \rightarrow_{u} y=(x \vee y) \rightarrow_{u} y \geq y$. Thus $y, x \vee y$, $(x \vee y) \rightarrow_{y} y$ and $(x \vee y) \rightarrow_{u} y$ all lie iin the Heyting algebra $y \uparrow$. The maximal element characterization of both $(x \vee y) \rightarrow_{y} y$ and $(x \vee y) \rightarrow_{u} y$ forces both to agree.

We will use the axioms of Heyting algebras to derive an axiomatization of skew Heyting algebras. The reader should find most of the axioms of Theorem 3.2 below to be intuitively clear generalizations to the non-commutative case. However, two axioms should be given further explanation. Firstly, the $u$ in axiom (SH4) below appears since upon passing to the non-commutative case, an element that is both below $x$ and $y$ with respect to the partial order $\leq$ no longer need exist. (We can have $x \wedge y \wedge x \leq x$ but not $x \wedge y \wedge x \leq y$ in general.) Similarly, axiom (SH0) is needed since in the non-commutative case it no longer follows from the other axioms, the reason being that in general $x \leq y \vee x \vee y$ need not hold.

Theorem 3.2. Let $(S ; \wedge, \vee, \rightarrow, 1)$ be an algebra of type $(2,2,2,0)$ such that $(S ; \wedge, \vee, 1)$ is a co-strongly distributive skew lattice with top 1 . Then $(S ; \wedge, \vee, \rightarrow, 1)$ is a skew Heyting algebra if and only if it satisfies the following axioms:

(SH0) $x \rightarrow y=(y \vee x \vee y) \rightarrow y$.

(SH1) $x \rightarrow x=1$,

(SH2) $x \wedge(x \rightarrow y) \wedge x=x \wedge y \wedge x$,

(SH3) $y \wedge(x \rightarrow y)=y$ and $(x \rightarrow y) \wedge y=y$,

(SH4) $x \rightarrow(u \vee(y \wedge z) \vee u)=(x \rightarrow(u \vee y \vee u)) \wedge(x \rightarrow(u \vee z \vee u))$.

Proof. Assume that $\mathbf{S}$ is a skew Heyting algebra.

(SH0). Both $x \rightarrow y$ and $(y \vee x \vee y) \rightarrow y$ are defined as $(y \vee x \vee y) \rightarrow_{y} y$. Thus they are equal.

(SH1). This is true because $1 \wedge x=x$ is true in $x \uparrow$. 


\section{Hence}

(SH2). In $y \uparrow(\mathrm{H} 2)$ implies $(y \vee x \vee y) \wedge\left((y \vee x \vee y) \rightarrow_{y} y\right)=(y \vee x \vee y) \wedge y=y$.

$$
x \wedge(y \vee x \vee y) \wedge(x \rightarrow y) \wedge x=x \wedge y \wedge x
$$

On the other hand,

$x \wedge(y \vee x \vee y) \wedge(x \rightarrow y) \wedge x=x \wedge(y \vee x \vee y) \wedge x \wedge(x \rightarrow y) \wedge x=x \wedge(x \rightarrow y) \wedge x$,

where we have used the regularity of $\wedge$ and the fact that $x \preceq y \vee x \vee y$.

(SH3). Both identities hold because $y \wedge(y \vee x \vee y)=y$ in $y \uparrow$. Thus $x \rightarrow y=$ $(y \vee x \vee y) \rightarrow y \geq y$.

(SH4). First note that (SH4) is equivalent to

(SH4') $(u \vee x \vee u) \rightarrow(u \vee(y \wedge z) \vee u)=((u \vee x \vee u) \rightarrow(u \vee y \vee u)) \wedge((u \vee x \vee u) \rightarrow$ $(u \vee z \vee u))$.

Indeed, (SH0) and the conormality of $\vee$ give both

$$
(u \vee x \vee u) \rightarrow(u \vee w \vee u)=(u \vee x \vee w \vee u) \rightarrow(u \vee w \vee u)
$$

and

$$
x \rightarrow(u \vee w \vee u)=(u \vee x \vee w \vee u) \rightarrow(u \vee w \vee u)
$$

so that

$$
x \rightarrow(u \vee w \vee u)=(u \vee x \vee u) \rightarrow(u \vee w \vee u)
$$

Hence it suffices to prove that (SH4') holds.

Observe that distributivity implies

$$
(u \vee y \vee u) \wedge(u \vee z \vee u)=u \vee(y \wedge z) \vee u
$$

Since $u \vee x \vee u, u \vee y \vee u, u \vee z \vee u$ and $u \vee(y \wedge z) \vee u$ all lie in $u \uparrow$ we can simply compute in $u \uparrow$. Using (3.1) and axiom (H4) for Heyting algebras we obtain: $(u \vee x \vee u) \rightarrow$ $(u \vee(y \wedge z) \vee u)=(u \vee x \vee u) \rightarrow((u \vee y \vee u) \wedge(u \vee z \vee u))=((u \vee x \vee u) \rightarrow$ $(u \vee y \vee u)) \wedge((u \vee x \vee u) \rightarrow(u \vee z \vee u))$

To prove the converse assume that (SH0)-(SH4) hold. Given arbitrary $u \in S$ and $x, y, z \in u \uparrow$ set $x \rightarrow_{u} y=x \rightarrow y$. Axiom (SH3) implies that $x \rightarrow y \in y \uparrow \subseteq u \uparrow$. Thus the restriction $\rightarrow{ }_{u}$ of $\rightarrow$ to $u \uparrow$ is well defined. Since $u \uparrow$ is commutative with bottom $u$, axioms (SH1)-(SH4) for $\rightarrow$ respectively simplify to (H1)-(H4) for $\rightarrow_{u}$, making $\rightarrow_{u}$ the Heyting implication on $u \uparrow$. Axiom (SH0) assures that $\rightarrow$ is indeed the skew Heyting implication satisfying $a \rightarrow b=(b \vee a \vee b) \rightarrow_{b} b$, for any $a, b \in S$.

Corollary 3.3. Skew Heyting algebras form a variety.

In the remainder of the paper, given a skew Heyting algebra we shall simplify the notation $\rightarrow_{u}$ to $\rightarrow$ when referring to the Heyting implication in $u \uparrow$. Remarks made about Heyting algebras in Section 2 apply here also. Given a co-strongly distributive skew lattice $(S ; \wedge, \vee, 1)$ with a top 1 , if a binary operation $\rightarrow$ exists that makes $(S ; \wedge, \vee, \rightarrow, 1)$ a skew Heyting algebra, then it is unique since it is already there implicitly. Hence, given two isomorphic skew lattices, if either is the reduct of a skew Heyting algebra, then so is the other and both are isomorphic as skew Heyting algebras.

Proposition 3.4. The relation $\mathcal{D}$ defined in (2.1) is a congruence on any skew Heyting algebra. 
Proof. Let $(S ; \wedge, \vee, \rightarrow, 1)$ be a skew Heyting algebra. Since $\mathcal{D}$ is a congruence for costrongly distributive skew lattices with a top, we only need to prove $(a \rightarrow b) \mathcal{D}(c \rightarrow d)$ holds for any $a, b, c, d \in S$ satisfying $a \mathcal{D} c$ and $b \mathcal{D} d$. Without loss of generality we may assume $b \leq a$ and $d \leq c$. (Otherwise replace $a$ by $b \vee a \vee b$ and $c$ by $d \vee c \vee d$.)

To begin, define a map $\varphi: b \uparrow \rightarrow d \uparrow$ by setting $\varphi(x)=d \vee x \vee d$. We claim that $\varphi$ is a lattice isomorphism of $(b \uparrow ; \wedge, \vee)$ with $(d \uparrow ; \wedge, \vee)$, with inverse $\psi: d \uparrow \rightarrow b \uparrow$ given by $\psi(y)=b \vee y \vee b$. It is easily seen that $\varphi$ and $\psi$ are inverses of each other. For instance, $\psi(\varphi(x))=b \vee d \vee x \vee d \vee b$ equals $(b \vee d \vee b) \vee x \vee(b \vee d \vee b)$ by the regularity of $\vee$. But the latter reduces to $b \vee x \vee b$ because $b \mathcal{D} d$, and since $x \in b \uparrow$ it reduces further to $x$ by Lemma 2.1, giving $\psi(\varphi(x))=x . \varphi$ must preserve $\wedge$ and $\vee$. Indeed distributivity gives:

$$
\varphi\left(x \wedge x^{\prime}\right)=d \vee\left(x \wedge x^{\prime}\right) \vee d=(d \vee x \vee d) \wedge\left(d \vee x^{\prime} \vee d\right)=\varphi(x) \wedge \varphi\left(x^{\prime}\right)
$$

And the regularity gives:

$$
\varphi\left(x \vee x^{\prime}\right)=d \vee\left(x \vee x^{\prime}\right) \vee d=(d \vee x \vee d) \vee\left(d \vee x^{\prime} \vee d\right)=\varphi(x) \vee \varphi\left(x^{\prime}\right)
$$

Thus $\varphi($ and $\psi$ ) is a lattice isomorphism of $b \uparrow$ with $d \uparrow$. But then $\varphi$ and $\psi$ are also isomorphisms of Heyting algebras. That is, e.g., $\varphi(x \rightarrow y)=\varphi(x) \rightarrow \varphi(y)$.

Next, observe that $x \mathcal{D} \varphi(x)$ for all $x \in b \uparrow$. Indeed, regularity gives:

$$
\varphi(x) \vee x \vee \varphi(x)=(d \vee x \vee d) \vee x \vee(d \vee x \vee d)=d \vee x \vee d=\varphi(x)
$$

and likewise $x \vee \varphi(x) \vee x=\psi(\varphi(x)) \vee \varphi(x) \vee \psi(\varphi(x))=\psi(\varphi(x))=x$. There is more: $a$ is the unique element in its $\mathcal{D}$-class belonging to $b \uparrow$ and $c$ is the unique element in the same $\mathcal{D}$-class belonging to $d \uparrow$ (since each upset $u \uparrow$ intersects any $\mathcal{D}$-class in at most one element). But $\varphi(a)$ in $d \uparrow$ behaves in the manner just like $c$, and so $\varphi(a)=c$. Since $b \mathcal{D} d, \varphi(b)=d \vee b \vee d=d$ and $\varphi(a \rightarrow b)=\varphi(a) \rightarrow \varphi(b)=c \rightarrow d$, thus giving $a \rightarrow b \mathcal{D} c \rightarrow d$.

Following [5] a commutative subset of a symmetric skew lattice is a non-empty subset whose elements both join and meet commute.

Theorem 3.5. Given a co-strongly distributive skew lattice $(S ; \wedge, \vee, 1)$ with top 1 , an operation $\rightarrow$ can be defined on $S$ making $(S ; \wedge, \vee, \rightarrow, 1)$ a skew Heyting algebra if and only if the operation $\rightarrow$ can be defined on $S / \mathcal{D}$ making $\left(S / \mathcal{D} ; \wedge, \vee, \rightarrow, \mathcal{D}_{1}\right)$ a generalized Heyting algebra.

Proof. To begin, for any $u$ in $S$, the upset $u \uparrow$ is a $\mathcal{D}$-class transversal of the principal filter $S \vee u \vee S$. Secondly, the induced homomorphism $\varphi: S \rightarrow S / \mathcal{D}$ is bijective on any commutative subset of $S$ since distinct commuting elements of $S$ lie in distinct $\mathcal{D}$-classes. It follows that for each $u$ in $S, \varphi$ restricts to an isomorphism of upsets, $\varphi_{u}: u \uparrow \cong \varphi(u) \uparrow$. Thus each upset $u \uparrow$ in $S$ forms a Heyting algebra if and only if each upset $v \uparrow$ in $S / \mathcal{D}$, being some $\varphi(u) \uparrow$, must form a Heyting algebra. The theorem follows.

Comment. This result is a near-dual of the important fact that a strongly distributive skew lattice $\mathbf{S}$ with bottom 0 is the (necessarily unique) reduct of a skew Boolean algebra if and only if its lattice image $\mathbf{S} / \mathcal{D}$ is the reduct of a (necessarily unique) generalized Boolean algebra. ([15], 3.8.) 
We next consider consequences of the above theorem. The first is on the "skew lattice side" of things and the next is more on the "Heyting side". But first recall the definitions of Green's relations $\mathcal{L}$ and $\mathcal{R}$ on a skew lattice:

$$
\begin{aligned}
& x \mathcal{L} y \Leftrightarrow(x \wedge y=x \& y \wedge x=y, \text { or equivalently } x \vee y=y \& y \vee x=x), \\
& x \mathcal{R} y \Leftrightarrow(x \wedge y=y \& y \wedge x=x, \text { or equivalently } x \vee y=x \& y \vee x=y) .
\end{aligned}
$$

Relations $\mathcal{L}$ and $\mathcal{R}$ are contained in the Green's relation $\mathcal{D}$ defined above and $\mathcal{L} \circ \mathcal{R}=$ $\mathcal{R} \circ \mathcal{L}=\mathcal{D}$ holds. A skew lattice is called right-handed if the relation $\mathcal{L}$ is trivial, in which case $\mathcal{D}=\mathcal{R}$, and it is called left-handed if the relation $\mathcal{R}$ is trivial, in which case $\mathcal{D}=\mathcal{L}$. By Leech's Second Decomposition Theorem [13] the relations $\mathcal{L}$ and $\mathcal{R}$ are congruences on any skew lattice $\mathbf{S}, \mathbf{S} / \mathcal{R}$ is left-handed, $\mathbf{S} / \mathcal{L}$ is right-handed and the following diagram is a pullback:

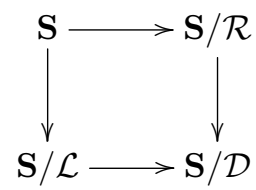

Corollary 3.6. If $\mathbf{S}=(S ; \wedge, \vee, 1)$ be a co-strongly distributive skew lattice with top 1 , then the following are equivalent:

1. $\mathbf{S}$ is the reduct of a skew Heyting algebra $(S ; \wedge, \vee, \rightarrow, 1)$.

2. $\mathbf{S} / \mathcal{L}$ is the reduct of a skew Heyting algebra $(S / \mathcal{L} ; \wedge, \vee, \rightarrow, 1)$.

3. $\mathbf{S} / \mathcal{R}$ is the reduct of a skew Heyting algebra $(S / \mathcal{R} ; \wedge, \vee, \rightarrow, 1)$.

Moreover, both $\mathcal{L}$ and $\mathcal{R}$ are congruences on any skew Heyting algebra.

Proof. The equivalence of (i)-(iii) is due to the preceding theorem plus the fact that $\mathbf{S} / \mathcal{D}$, $(\mathbf{S} / \mathcal{L}) / \mathcal{D}_{S / \mathcal{L}}$ and $(\mathbf{S} / \mathcal{R}) / \mathcal{D}_{S / \mathcal{R}}$ are isomorphic. Next, the induced map $\rho: \mathbf{S} \rightarrow \mathbf{S} / \mathcal{L}$ is at least a homomorphism of co-strongly distributive skew lattices. By the argument of the preceding theorem, it induces isomorphisms between corresponding pairs of upsets, $u \uparrow$ in $S$ and $\mathcal{L}_{u} \uparrow$ in $S / \mathcal{L}$. Thus given $x \rightarrow y=(y \vee x \vee y) \rightarrow_{y} y$ and $u \rightarrow v=(v \vee u \vee v) \rightarrow_{v} v$ with $x ;, \mathcal{L} ;, u$ and $y ;, \mathcal{L} ;, v$ in $S$, both $(y \vee x \vee y) \rightarrow_{y} y$ and $(v \vee u \vee v) \rightarrow_{v} v$ are mapped to the common $\mathcal{L}_{y \vee x \vee y} \rightarrow \mathcal{L}_{y} \mathcal{L}_{y}$, making $x \rightarrow y ;, \mathcal{L} ;, y \rightarrow v$ in $\mathbf{S}$. A similar argument applies to the induced map $\lambda: \mathbf{S} \rightarrow \mathbf{S} / \mathcal{R}$.

An alternative to the characterization of Theorem 3.2 is given by:

Corollary 3.7. Every skew Heyting algebra satisfies:

(SHA) $x \preceq y \rightarrow z$ if and only if $x \wedge y \preceq z$.

In particular, $x \rightarrow y=1$ iff $x \preceq y$.

In general, an algebra $\mathbf{S}=(S ; \wedge, \vee, \rightarrow, 1)$ of type $(2,2,2,0)$ is a skew Heyting algebra if the following conditions hold:

1. The reduct $(S ; \wedge, \vee, 1)$ is a co-strongly distributive skew lattice with top 1 .

2. $y \leq x \rightarrow y$ holds for all $x, y \in S$. 


\section{S satisfies axiom (SHA).}

Proof. Given that $S$ is a skew Heyting algebra, since the induced epimorphism $\varphi: S \rightarrow$ $S / \mathcal{D}$ is a homomorphism of skew Heyting algebras we have

$$
x \preceq y \rightarrow z \text { iff } \varphi(x) \leq \varphi(y) \rightarrow \varphi(z) \text { iff } \varphi(x) \wedge \varphi(y) \leq \varphi(z) \text { iff } x \wedge y \preceq z .
$$

Conversely, let $\mathbf{S}=(S ; \wedge, \vee, \rightarrow, 1)$ be an algebra of type $(2,2,2,0)$ satisfying (1)-(3). Suppose that $x, y, z$ lie in a common upset $u \uparrow$. Since $\preceq$ is just $\leq$ in $u \uparrow$ nad $y \rightarrow z$ lies in $u \uparrow$ by (2) we have $x \leq y \rightarrow z$ iff $x \wedge y \leq z$ in $u \uparrow . \quad(S ; \wedge, \vee, 1)$ is thus at least a skew Heyting lattice. Now consider the derived implication $\rightarrow^{*}$ given by $x \rightarrow^{*} y=$ $(y \vee x \vee y) \rightarrow_{y} y$. Both $y \rightarrow z$ and $y \rightarrow^{*} z$ satisfy (SHA) and thus are $\mathcal{D}$-equivalent. But since both lie in the sublattice $z \uparrow$, they must be equal.

We have seen that each skew Heyting algebra is basically a co-strongly distributive skew lattice $\mathbf{S}$ with top, say 1 , for which $\mathbf{S} / \mathcal{D}$ is a generalized Heyting algebra, in which case the Heyting structure on each upset $u \uparrow$ of $\mathbf{S}$ is obtained from that of the isomorphic upset $\mathcal{D}_{u} \uparrow$ in $\mathbf{S} / \mathcal{D}$. This suggests that all standard classes of generalized Heyting algebras yield classes of skew Heyting algebras whose maximal commutative images belong to the particular class. We consider several cases.

Case 1. Finite distributive lattices possess a well-defined Heyting algebra structure. Thus any finite co-strongly distributive skew lattice with a top, or more generally any co-strongly distributive skew lattice with a top and a finite maximal lattice image is the reduct of a unique skew Heyting algebra.

Case 2. All chains possessing a top 1 form Heyting algebras. Here things are simple:

$$
x \rightarrow y= \begin{cases}1 ; & \text { if } x \leq y \\ y ; & \text { otherwise }\end{cases}
$$

Thus all co-strongly distributive skew chains with a top are skew Heyting algebra reducts, where a skew chain is any skew lattice $\mathbf{S}$ where $\mathbf{S} / \mathcal{D}$ is a chain, i.e., $\preceq$ is a total preorder on $\mathbf{S}$. Here, given $x, y$ in a common $u \uparrow$ one has:

$$
x \rightarrow y= \begin{cases}1 ; & \text { if } x \preceq y . \\ y ; & \text { otherwise }\end{cases}
$$

Case 3. Dual generalized Boolean algebras. These are algebras $\mathbf{S}=(S ; \wedge, \vee, \backslash \backslash, 1)$ where $(S ; \wedge, \vee, 1)$ is a distributive lattice with top 1 and $\backslash \backslash$ is a binary operation on $S$ such that $(y \vee x) \vee(y \backslash \backslash x)=1$ and $(y \vee x) \wedge(y \backslash \backslash x)=y$ for all $x, y$ in $S$. As with $\backslash$ for generalized Boolean algebras, $\backslash$ is uniquely determined. Moreover, in this case, $x \rightarrow y=y \backslash x$. A dual-skew Boolean algebra $\mathbf{S}=(S ; \wedge, \vee, \backslash \backslash, 1)$ is an algebra such that $(S ; \wedge, \vee, 1)$ is a co-strongly distributive skew lattice with top 1 and binary operation $\backslash$ such that:

$$
\begin{aligned}
& (y \vee x \vee y) \vee(y \backslash \backslash x)=1=(y \backslash x) \vee(y \vee x \vee y) \\
& (y \vee x \vee y) \wedge(y \backslash \backslash x)=y=(y \backslash \backslash x) \wedge(y \vee x \vee y)
\end{aligned}
$$


The relevant diagram is:

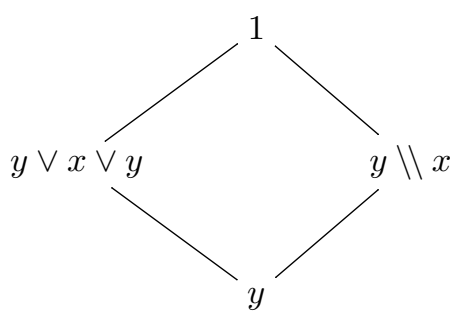

These dual algebras are, of course, precisely the co-strongly distributive skew lattices with a top whose maximal lattice images are the lattice reducts of dual-generalized Boolean algebras. Once again we follow the commutative case: $x \rightarrow y=y \| x$ which now is $y \backslash(y \vee x \vee y)$ in $y \uparrow$.

We thus have:

Corollary 3.8. A co-strongly distributive skew lattice with a top $\mathbf{S}=(S ; \wedge, \vee, 1)$ is the reduct of a uniquely determined skew Heyting algebra $(S ; \wedge, \vee, \backslash, 1)$ if any one of the following conditions holds:

1. $\mathbf{S} / \mathcal{D}$ is finite.

2. $\mathbf{S}$ is a skew chain.

3. $\mathbf{S}$ is the reduct of a dual generalized Boolean algebra, $\mathbf{S}=(S ; \wedge, \vee, \backslash \backslash, 1)$.

Implicit in Case 3 is a basic duality that occurs for skew lattices. Given a skew lattice $\mathbf{S}=(S ; \wedge, \vee)$, its (vertical) dual is the skew lattice $\mathbf{S}^{\uparrow}=(S ; \wedge \uparrow, \vee \uparrow)$, where as binary functions, $\wedge^{\uparrow}=\vee$ and $\vee \downarrow=\wedge$. Clearly $\mathbf{S}^{\uparrow \uparrow}=\mathbf{S}$ and any homomorphism $f: \mathbf{S} \rightarrow \mathbf{T}$ of skew lattices ia also a homomorphism from $\mathbf{S}^{\mathfrak{}}$ to $\mathbf{T}^{\mathfrak{}}$; moreover a skew lattice $\mathbf{S}$ is distributive (or symmetric) iff $\mathbf{S}^{\uparrow}$ is thus. Either $\mathbf{S}$ or $\mathbf{S}^{\uparrow}$ is strongly distributive iff the other is co-strongly distributive; more generally, $\mathbf{S}$ or $\mathbf{S} \uparrow$ is normal iff the other is co-normal. Also, one has a bottom element iff the other has a top element, both being the same element in $S$.

Expanding the signature, $(S ; \wedge, \vee, \backslash, 0)$ is a skew Boolean algebra if and only if its dual $\left(S ; \wedge^{\uparrow}, \vee \uparrow, \backslash, 1\right)$ is a dual skew Boolean algebra where $\backslash$ and 0 are replaced by $\backslash$ and 1 . Thus any skew Boolean algebra $(S ; \wedge, \vee, \backslash, 0)$ induces a skew Heyting algebra $\left(S ; \wedge^{\uparrow}, \vee \uparrow, \rightarrow, 1\right)$ where $x \rightarrow y=y \backslash x$ and $1=$ old 0 . Standard examples of skew Boolean algebras thus give us:

Example 3.9. Given sets $X$ and $Y$, the skew Heyting operations derived from the skew Boolean operations on the set $\mathcal{P}(X, Y)$ of all partial functions from $X$ to $Y$ are as follows.

\begin{tabular}{|c|c|c|}
\hline skew Heyting operation & description & skew Boolean operation \\
\hline$f \wedge g$ & $f \cup\left(\left.g\right|_{\operatorname{dom} g-\operatorname{dom} f}\right)$ & $f \vee g$ \\
$f \vee g$ & $\left.g\right|_{\operatorname{dom} g \cap \operatorname{dom} f}$ & $f \wedge g$ \\
$f \rightarrow g$ & $\left.g\right|_{\operatorname{dom} g-\operatorname{dom} f}$ & $g \backslash f$ \\
1 & $\emptyset$ & 0 \\
\hline
\end{tabular}


Example 3.10. Given a surjective function $\pi: Y \rightarrow X$, let $\operatorname{Sec}(\pi)$ denote the set of all sections of $\pi$, that is, functions $f$ from subsets $U$ of $X$ to $Y$ such that $\pi \circ f=i d_{\operatorname{dom}(f)}$. Skew Heyting algebra operations and corresponding skew Boolean operations are defined on $\operatorname{Sec}(\pi)$ using precisely the above descriptions. At first glance this seems to be just a subalgebra of the type of algebra in Example 1. The latter, however, is isomorphic to $\operatorname{Sec}(\pi)$ where $\pi$ is now the coordinate projection of $X \times Y$ onto $X$. Modifications of this example arise in the dualities of the next section.

It so happens that any right-handed (co-)strongly distributive skew lattice is isomorphic to a subset of partial functions in some $\mathcal{P}(X, Y)$ that is closed under the relevant $\wedge$ and $\vee$ operations above. (See [12] Section 3.7.) It follows that the skew lattice reduct of a skew Heyting algebra is isomorphic to some such partial function algebra. The difference of this more general case from that of the two examples above is that here $x \rightarrow y$ need not have a polynomial definition, unlike the co-Boolean case where $x \rightarrow y=y \backslash x$.

The following result is useful for computing in skew Heyting algebras.

Proposition 3.11. Let $\mathbf{S}=(S ; \wedge, \vee, \rightarrow, 1)$ be a skew Heyting algebra and $x, y, z \in S$. Then

$$
(x \vee y \vee x) \rightarrow z=(x \rightarrow z) \wedge(y \rightarrow z) \wedge(x \rightarrow z)
$$

Proof. As $\mathbf{S} / \mathcal{D}$ is a generalized Heyting algebra and relation $\mathcal{D}$ respects all skew Heyting algebra operations, it follows that $(x \vee y \vee x) \rightarrow z \mathcal{D}(x \rightarrow z) \wedge(y \rightarrow z) \wedge(x \rightarrow z)$. However, both $(x \vee y \vee x) \rightarrow z$ and $(x \rightarrow z) \wedge(y \rightarrow z) \wedge(x \rightarrow z)$ are above $z$ with respect to the natural partial order, and hence must be equal by Lemma 2.2.

A skew lattice $\mathbf{S}$ is meet [join] complete if each nonempty commutative subset possesses an infimum [a supremum] in $S$. It follows from the dual of [5] Proposition 2.10 that if $\mathbf{S}$ is a meet complete co-strongly distributive skew lattice with 1 , then $\mathbf{S}$ is complete. We call a skew Heyting algebra complete if it is complete as a skew lattice.

\section{Connections to duality}

Dual skew Boolean algebras are order duals (upside-downs) to usually studied skew Boolean algebras. Skew Boolean algebras and dual skew Boolean algebras are categorically isomorphic. Right-handed (dual) skew Boolean algebras are dually equivalent to sheaves over locally compact Boolean spaces by results of [1] and [12], where a locally compact Boolean space is a topological space whose one-point-compactification is a Boolean space. The obtained duality asserts that any right- [left-]handed skew Boolean algebra is isomorphic to the skew Boolean algebra of compact open sections (i.e. sections over compact open subsets) of an étale map over some locally compact Boolean space. Let us note that the restriction to right- [left-]handed algebras is not a major restriction since Leech's Second Decomposition Theorem yields that any skew lattice is a pull back of a left-handed and a right-handed skew lattice over their common maximal lattice image [13]. The general two-sided case was also covered in [1].

Bounded distributive lattices are dual to Priestley spaces; in this duality each bounded distributive lattice is represented as the distributive lattice of all clopen (i.e. closed and open) upsets of a Priestley space. The Esakia duality established in [9] yields that Heyting algebras are dual to Esakia spaces, i.e. those Priestley spaces in which the downset of each 
clopen set is again clopen. Moreover, if $(X, \leq, \tau)$ is an Esakia space then given clopen subsets $U$ and $V$ in $X$ the implication is defined by

$$
U \rightarrow V=X \backslash \downarrow(U \backslash V) .
$$

Duality for strongly distributive skew lattices was recently established in [2]. It yields that right-handed strongly distributive skew lattices with bottom are dual to the sheaves over locally Priestley spaces, where by a locally Priestley space we mean an ordered topological space whose one-point-compactification is a Priestley space. Via the obtained duality each right-handed strongly distributive skew lattice with bottom is represented as a skew lattice of sections over copen (i.e. compact and open) downsets of a locally Priestley space, with the operations being defined as follows:

$$
\begin{aligned}
0 & =\emptyset, \\
r \wedge s & =\left.s\right|_{\operatorname{dom} r \cap \operatorname{dom} s}, \\
r \vee s & =\left.r \cup s\right|_{\operatorname{dom} s-\operatorname{dom} r}, \\
r \backslash s & =\left.r\right|_{\operatorname{dom} r-\operatorname{dom} s} .
\end{aligned}
$$

Given a distributive lattice $\mathbf{L}$ denote by $\mathbf{L}^{c}$ the distributive lattice that is obtained from $\mathbf{L}$ by reversing the order. Denote by DL the category of all distributive lattices, by PS the category of all locally Priestley spaces and consider the functors:

$$
\begin{aligned}
{ }^{c}: \mathbf{D L} & \rightarrow \mathbf{D L} \\
\mathbf{L} & \mapsto \mathbf{L}^{c}
\end{aligned} \quad \text { and } \quad{ }^{\prime}: \begin{array}{cl}
\mathbf{P S} & \rightarrow \mathbf{P S} \\
(X, \leq) & \mapsto(X, \geq) .
\end{array}
$$

Restricting the functors ${ }^{c}$ and $r$ to the categories HA of all Heyting algebras and ES of all Esakia spaces, respectively, yields the following isomorphism of categories:

$$
\begin{aligned}
{ }^{c}: \mathbf{H A} & \rightarrow \mathbf{c H A} \\
\mathbf{L} & \mapsto \mathbf{L}^{c}
\end{aligned} \quad \text { and } \quad r^{\prime} \quad \begin{array}{cl}
\mathbf{E S} & \rightarrow \mathbf{c E S} \\
(X, \leq) & \mapsto(X, \geq),
\end{array}
$$

where cHA denotes the category of all co-Heyting algebras (defined as order-inverses of Heyting algebras) and cES denotes the category of all co-Esakia spaces the latter being introduced in [3] as Priestley spaces in which an upset of a clopen is again clopen.

We introduce the following categories:

SDSL : strongly distributive skew lattices with 0 ,

cSDSL : co-strongly distributive skew lattices with 1,

SHA : skew Heyting algebras,

cSHA : co-skew Heyting algebras,

with the latter being defined as the category of all algebras of the form $\mathbf{S}^{c}$, where $\mathbf{S}$ is a skew Heyting algebra and

$$
\begin{aligned}
{ }^{c}: \text { SDSL } & \rightarrow \text { cSDSL } \\
\mathbf{S} & \mapsto \mathbf{S}^{c}
\end{aligned}
$$

is the isomorphism of categories that turns a skew lattice upside-down. The restriction of the functor ${ }^{c}$ to the categories CSHA and SHA yields the isomorphism:

$$
\begin{aligned}
{ }^{c}: \text { CSHA } & \rightarrow \text { SHA } \\
\mathbf{S} & \mapsto \mathbf{S}^{c}
\end{aligned}
$$


The isomorphism of categories induces an isomorphism of concepts:

\begin{tabular}{|c|c|}
\hline SHA & cSHA \\
\hline$\wedge$ & $\vee$ \\
$\vee$ & $\wedge$ \\
1 & 0 \\
strong codistributivity & strong distributivity \\
filter & ideal \\
prime filter & prime ideal \\
\hline
\end{tabular}

It follows from Theorem 3.5 that the skew Heyting algebra structure can be imposed exactly on those co-strongly distributive skew lattices with top whose maximal lattice image is a generalized Heyting algebra. Therefore the duality for right-handed skew Heyting algebras yields that they are dual to sheaves over local Esakia spaces, i.e. ordered topological spaces whose one-point-compactification is an Esakia space.

Let $(B, \leq)$ be an Esakia space, $E$ a topological space and $p: E \rightarrow B$ a surjective étale map. Consider the set $S$ of all sections of $p$ over copen upsets in $B$, i.e. an element of $S$ is a map $s: U \rightarrow E$, where $U$ is a copen upset in $B$, that satisfies the property $p \circ s=\mathrm{id}_{U}$. A section $s \in S$ is considered to be below a section $r \in S$ when $s$ extends $r$. The skew Heyting operations are defined on $S$ by:

$$
\begin{array}{r}
r \vee s=\left.s\right|_{\operatorname{dom} r \cap \operatorname{dom} s}, \\
r \wedge s=\left.r \cup s\right|_{\operatorname{dom} s \backslash \operatorname{dom} r}, \\
r \rightarrow s=\left.r\right|_{\uparrow(\operatorname{dom} \backslash \backslash \operatorname{dom} r)} \\
1=\emptyset .
\end{array}
$$

Theorem 4.1. Let $p: E \rightarrow B$ be a surjective étale map over a local Esakia space B. Then the set $S$ of all sections of $p$ over copen upsets in $B$ forms a skew Heyting algebra under the above operations.

\section{References}

[1] A. Bauer and K. Cvetko-Vah, Stone duality for skew Boolean algebras with intersections, Houston J. Math. 39 (2013), 73-109.

[2] A. Bauer, K. Cvetko-Vah, M. Gehrke, S. van Gool and G. Kudryavtseva, A Non-Commutative Priestley Duality, Topology and Appl. 160 (2013), 1423-1438.

[3] G. Bezhanishvili, N. Bezhanishvili, D. Gabelaia and A. Kurz, Bitopological duality for distributive lattices and Heyting algebras, Math. Structures Comput. Sci. 20 (2010), 359-393.

[4] N. Bezhanishvili, Lattices of Intermediate and Cylindric Modal Logics, PhD Thesis, ILLC, University of Amsterdam, 2006.

[5] R. J. Bignall and J. E. Leech, Skew Boolean algebras and discriminator varieties, Alg. Universalis 33 (1995), 387-398.

[6] R. J. Bignall and M. Spinks, Some New Strategies for Theorem-Proving in First Order Logic with Equality, Proc. ISMVL 1997/98.

[7] R. J. Bignall, M. Spinks and R. Veroff, Discriminator logics (Research announcment), Australasian Journal of Logic (11:2) (2014), 159-171. 
[8] J. P. Pita da Costa, On the Coset Structure of a Skew Lattice, Demonstratio Mathematica XLIV No. 4 (2011), 673-692.

[9] L. L. Esakia, Topological Kripke models, Soviet Mathematics Doklady 15 (1974), 147-151.

[10] M. Gehrke, Canonical extensions, Esakia spaces, and universal models, Chapter in Leo Esakia on duality in modal and intuitionistic logics, upcoming special volume in the series Trends in Logic (Outstanding Contributions subseries) dedicated to the achievements of Leo Esakia, 2014.

[11] P. Jordan, Über nichtkommutative Verbände, Arch. Math. 2 (1949), 56-59.

[12] G. Kudryavtseva, A refinement of Stone duality to skew Boolean algebras, Algebra Universalis 67 (2012), 397-416.

[13] J. Leech, Skew lattices in rings, Alg. Universalis 26 (1989), 48-72.

[14] J. Leech, Skew Boolean Algebras, Alg. Universalis 27 (1990), 497-506.

[15] J. Leech, Normal skew lattices, Semigroup Forum 44 (1992), 1-8.

[16] H. A. Priestley, Representation of distributive lattices by means of ordered stone spaces, Bull. Lond. Math. Soc. 2 (1970), 186-190.

[17] H. A. Priestley, Ordered topological spaces and the representation of distributive lattices, Proc. Lond. Math. Soc. (3) 24 (1972), 507-530.

[18] B. M. Schein, Pseusosemilattices and Pseudolattices, Amer. Math. Soc. Transl. (2) 119 (1983), $1-16$.

[19] M. Spinks and R. Veroff, Axiomatizing the skew Boolean propositional calculus, J. Automat. Reason. 37 (2006), no. 1-2, 3-20.

[20] M. H. Stone, The theory of representation for Boolean algebras, Trans. Amer. Math. Soc. 74 (1936), 37-111. 